\title{
Capacidad de Discriminación de Dos Redes de Sensores como Unidades Sensibles de una Mini-Lengua Electrónica sobre Muestras de Leche
}

\author{
Álvaro Á. Arrieta ${ }^{(1) \star}$, Jorge A. Diaz ${ }^{(2)}$ y Manuel S. Palencia ${ }^{(3)}$ \\ (1) Universidad de Sucre, Departamento de Biología y Química, Carrera 28 \# 5-267 Barrio Puerta Roja, \\ Sincelejo, Colombia (e-mail: alvaroangel.arrieta@gmail.com) \\ (2) Universidad Pontificia Bolivariana, Laboratorio DANM, Grupo de desarrollo y aplicación de nuevos \\ materiales, Cra 6 № 97A-99, Montería, Colombia (e-mail: jarmandiaz1601@gmail.com) \\ (3) Universidad del Valle, Departamento de Química, Facultad de Ciencias Naturales y Exactas, Calle 13 \\ No 100-00, Cali-Colombia (e-mail: manuel.palencia@correounivalle.edu.co)
}

Recibido Mar. 6, 2017; Aceptado Abr. 25, 2017; Versión final Jun. 8, 2017, Publicado Oct. 2017

\begin{abstract}
Resumen
En este artículo se presentan los resultados de discriminación de dos redes de sensores. Una de estas redes de sensores se conformó por electrodos comerciales serigrafiados con materiales electroactivos y la otra red de sensores se elaboró a partir de electrodos modificados con polipirrol y diferentes dopantes. Se analizó la capacidad de discriminación y clasificación de diferentes tipos de muestras de leche comercial. Los resultados muestran que el uso de sensores modificados con polipirrol permite obtener respuestas más completas en información, clasificando correctamente las muestras de leche con propiedades fisicoquímicas y organolépticas diferentes mediante el uso de dos componentes principales que reprodujo el $67,6 \%$ de la información. Por otra parte, la red de sensores comerciales se evaluó sobre el mismo grupo de muestras e iguales condiciones, consiguiéndose el $56,5 \%$ de la variabilidad de información, mostrando así menos capacidad de discriminación de las muestras analizadas.
\end{abstract}

Palabras clave: polipirrol; electrodos serigrafiados; sensores electroquímicos; leche; lengua electrónica.

\section{Ability of Discrimination of Two Sensor Networks as Sensitive Units of a Mini-Electronic Tongue on Milk Samples}

\begin{abstract}
This paper presents the development of two sensor networks as initial stage for the design of a prototype electronic tongue. One of these sensor networks was joined by commercial screen printed electrodes and the other sensor network was assembled from modified electrodes with pyrrole and different dopants. The capacity of discrimination and classification of different types of samples of commercial milk to two sensor networks was analyzed. The results show that the use of modified sensors with conductive polymers allows obtaining more complete information, classifying correctly samples of milk with different organoleptic and physico-chemical properties by the use of two main components that reproduced the $67,6 \%$ of information. On the contrary, the commercial sensor network evaluated on the same group of samples and equal conditions, obtaining $56,5 \%$ of the variability of information, showing in this way lower classifying capacity of the samples analyzed.
\end{abstract}

Keywords: pyrrole; screen-printed electrodes; conductive polymers; milk; electronic tongue. 


\section{INTRODUCCIÓN}

Los sistemas electrónicos bioinspirados como lenguas electrónicas voltamétricas usan como elemento fundamental redes de sensores que acompañados de una técnica electroquímica como la voltametría cíclica y herramientas de análisis multivariante permiten recoger información para discriminar y clasificar sustancias complejas. (González-Calabuig et al., 2016; Huynh y Kutner 2015; Blanco et al., 2015; MariLuz 2016). Los primeros prototipos de lengua electrónica voltamétricas se basaron en la utilización de sensores metálicos de oro, iridio, platino, plata, cobre, níquel, cobalto, entre otros. (Campos et al., 2013; Lu et al., 2016). Sin embargo, el uso de algunos metales como elementos principales de captación de señales presentaban ciertas dificultades como la formación de capas de óxido cuando son manipulados a ciertos grados de $\mathrm{pH}$ y potencial redox. Adicionalmente esta oxidación y la presencia de iones o moléculas neutras pueden generar reacciones de formación de precipitados. De ahí que, estos sensores provocaran variaciones irregulares en la intensidad de corriente y por consiguiente ofrecen una discriminación deficiente de las muestras con respecto al tiempo de uso.

No obstante, el uso de especies químicas modificadoras de las superficies desnudas de los sensores metálicos como los polímeros conductores, permite la adquisición de información de manera eficiente de las características fisicoquímicas y organolépticas de las sustancias. Debido que un sensor modificado con una sustancia electroactiva como el polipirrol puede proporcionar señales producto de las especies químicas presentes en la muestra analizada y la interacción de su matriz con el material electroacivo en la superficie del sensor. (Paixão y Bertotti, 2009; Gutiérrez et al., 2013; Vagin y Winquist, 2015). De acuerdo a esta alternativa surgen sistemas electrónicos con mayor capacidad de clasificación y discriminación, que toman gran importancia para la vigilancia de la calidad de los alimentos. (Wei y Wang, 2013; Wey y Wang, 2011; Novakowsky et al., 2011; Dias et al., 2009).

Por tanto, el propósito de este estudio busca comparar la capacidad de discriminación y clasificación de muestras de leche, utilizando dos configuraciones de redes de sensores como unidades sensibles de una lengua electrónica. Específicamente se conformaron dos redes de sensores modificados con sustancias electroactivas, uno de ellos se construyó a partir de electrodos comerciales serigrafiados con materiales electroactivos y en el otro grupo, con sensores modificamos con polipirrol dopado con diferentes iones dopantes. El trabajo consistió en realizar medidas en muestras de leche con las dos redes de sensores y a partir de los resultados obtenidos por aplicación de análisis de componentes principales determinar que configuración sensorial ofrece mayores ventajas a la hora de hacer discriminación en muestras de leche.

\section{METODOLOGÍA}

Se analizaron en total 28 muestras; siete réplicas de cuatro muestras de leche comercial de diferentes clases (entera pasteurizada, entera, semidescremada deslactosada y semidescremada). Todos los reactivos utilizados fueron de grado analítico y se adquirieron de Sigma-Aldrich.

Para la red de sensores comerciales (red de sensores 1), fueron adquiridos de DropSens sensores serigrafiados (SPEs); integrados con un electrodo de referencia de plata, un electrodo de trabajo de material electroactivo según referencia y un contraelectrodo de carbón. En la tabla 1 se presenta la lista de los sensores y la naturaleza química del material electroactivo en el electrodo de trabajo. Para la fabricación de la red de sensor modificada con polipirrol (red de sensores 2) se utilizó: pirrol (Py), perclorato de litio (PL), sulfato de litio (SL), dodecilbencenosulfonato de sodio (DBS), perclorato de tetrabutil amonio (TBAP), persulfato de amonio (PSA) y ácido antraquinona 1,5-disulfónico hidrato de sal disódica (AQDD). Todos los reactivos se prepararon en agua ultra pura Milli-Q.

La red de sensores fue elaborada a partir de síntesis electroquímica en soluciones de $0.1 \mathrm{M}$ de pirrol y $0.2 \mathrm{M}$ de dopante, aplicando un potencial de $0.8 \mathrm{~V}$ y tiempo de polimerización de $100 \mathrm{~s}$. (Arrieta et al., 2016). Para conservar la estructura de la red de sensores, se utilizaron como sustratos los sensores comerciales de platino DRP-550 de DropSens con electrodo de referencia de plata y contraelectrodo de platino. Estos sensores fueron utilizados como sustratos y se depositó sobre la superficie del electrodo de trabajo (platino) películas de polipirrol con cada uno de los agentes dopantes mencionados anteriormente, con lo cual se construyó una red de seis sensores (tabla 1).

\section{Lengua electrónica y Registro de señales}

Como se mencionó anteriormente, con el fin de evaluar la capacidad de discriminación y clasificación de las redes de sensores con la lengua electrónica en muestras de leche se construyeron dos redes, la primera se le llamó RS1, la cual se ensambló a partir de 6 sensores comerciales serigrafiados con materiales electroactivos, los cuales fueron utilizados sin ninguna modificación o tratamiento adicional, el conjunto de sensores se presentan en la tabla 1. 
Tabla 1: Lista de sensores utilizados en las redes 1 y 2

\begin{tabular}{|l|l|l|l|}
\hline \multicolumn{2}{|c|}{ Red de sensores 1(RS1) } & \multicolumn{2}{c|}{ Red de sensores 2 (RS2) } \\
\hline Código & Electrodo trabajo (sensor) & Código & Electrodo trabajo (sensor) \\
\hline DS-710 & Azul de Prusia & PPy/PL & Polipirrol/perclorato de litio \\
\hline DS-410 & Ftalocianina de Cobalto & PPy/SL & Polipirrol/sulfato de litio \\
\hline DS-610 & Azul de meldola & PPy/DBS & $\begin{array}{l}\text { Polipirrol/ dodecillbencenosulfonato de } \\
\text { sodio }\end{array}$ \\
\hline DS-F10 & Ferrocianuro & PPy/TBAP & Polipirrol/ perclorato de tetrabutil amonio \\
\hline DRP-110PANI & Polianilina & PPy/PSA & Polipirrol/ persulfato de amonio \\
\hline DRP-110GPH & Grafeno & PPy/AQDD & $\begin{array}{l}\text { Polipirrol/ ácido antraquinona 1,5- } \\
\text { disulfónico }\end{array}$ \\
\hline
\end{tabular}

Por otro lado, la segunda red de sensores RS2 se elaboró a partir de pirrol y diferentes agentes dopantes tal como se describió anteriormente. Para realizar las medidas se utilizó una mini-lengua electrónica desarrollada en nuestros laboratorio, la cual está constituida por un dispositivo multicanal elaborado a partir de tecnología PSoC, el cual permite tener un dispositivo integrado en un chip y facilita su completa portabilidad debido a su pequeño tamaño $(2.8 \mathrm{~cm} \times 6 \mathrm{~cm} \times 9 \mathrm{~cm})$ y bajo peso $(120 \mathrm{~g})$. Los voltamogramas o señales registradas se visualizaron en un teléfono inteligente (Smartphone) Samsung Note 3 mediante una aplicación también diseñada en nuestros laboratorios que permite además la aplicación inmediata del Análisis de Componentes Principales. Detalles de este desarrollo han sido publicados previamente. (Amin y Arrieta, 2016).

Las medidas electroquímicas se llevaron a cabo mediante el registro de voltamogramas con las dos redes de sensores sobre cada una de las muestras de leche analizadas, el montaje de medida se ilustra en la figura 1., el cual corresponde a un dispositivo de mini-lengua electrónica de nuevo desarrollo, consistente en un sistema de registro portable, controlado desde un teléfono inteligente, el cual además permite realizar de manera automática el tratamiento estadístico. Los registros de las señales voltamétricas se obtuvieron aplicando un rango de potencial de -1 a $0,5 \mathrm{~V}$ y una velocidad de barrido de $0,1 \mathrm{~V} . \mathrm{s}-1$. Las medidas se realizaron a temperatura ambiente. Para obtener señales estables los sensores se ciclaron inicialmente 10 veces y luego se ejecutó un ciclo como medida final para alimentar la matriz de datos. Los sensores fueron descartados una vez la intensidad de la señal voltamétrica disminuía más de $10 \%$. Las medidas fueron realizadas en las muestras comerciales sin ningún tipo de pre-procesamiento y se realizaron de manera sucesiva con un lavado de las redes de sensores con agua destilada y posterior secado por 5 min entre muestras, cada medida tardó en promedio 10 min.

\section{Quimiometría}

Una vez registradas las señales se aplicó el análisis de componentes principales para reducir el número de variables y evaluar la capacidad de discriminación de las redes estudiadas. En cada caso se utilizaron 6 sensores, tomando como entrada los valores de corriente de las señales voltamétricas sin ningún tipo de pretratamiento. La matriz de valores que alimentó la PCA se construyó a partir de 28 filas (4 muestras de leche $x 7$ réplicas) y 9006 columnas ( 6 sensores x 1501 valores de corriente) que en total generaron una matriz de 252168 valores de corriente.

\section{RESULTADOS Y DISCUSIÓN}

En el desarrollo de lenguas electrónicas eficientes que permitan discriminar y clasificar sustancias complejas, es necesario conseguir sensores estables y que registren señales con selectividad cruzada que respondan a cada uno de las muestras y sus particularidades en cuanto a composición química y propiedades organolépticas colectando en las señales la mayor cantidad de información posible. (Rahaman y Kang, 2015; De Saja et al., 2006; Cetó et al., 2012; Ciosek y Wroblewski 2008; Hruskar et al., 2010; Elizabeth et al., 2011). En este sentido, se realizó un estudio preliminar para evaluar la estabilidad de las dos redes de sensores RS1 y RS2. Para llevar a cabo este estudio se utilizaron sensores nuevos en RS1 y recién preparados en RS2. Con cada red de sensores se realizaron medidas diariamente en una muestra de leche entera con un registro de 30 ciclos voltamétricos, hasta que se observara una variación superior al $10 \%$. Se encontró que la red de sensores RS1 después de aproximadamente 12 días de uso, las señales se tornaban completamente distorsionadas (con exceso de ruido) y en algunos de los sensores las cintas serigrafiadas (material electroactivo) se desprendieron de manera parcial del sustrato, afectando considerablemente el registro de 
las medidas y la estabilidad de las señales. Para el caso de la red de sensores RS2 no se observó el fenómeno de desprendimiento, pero si se evidenció perdida de la intensidad de las señales por desdopado de manera gradual. Después de 21 días de uso, el desdopado fue evidenciado con una pérdida de la intensidad de la señal por sobre el $10 \%$ en la mayoría de los sensores.

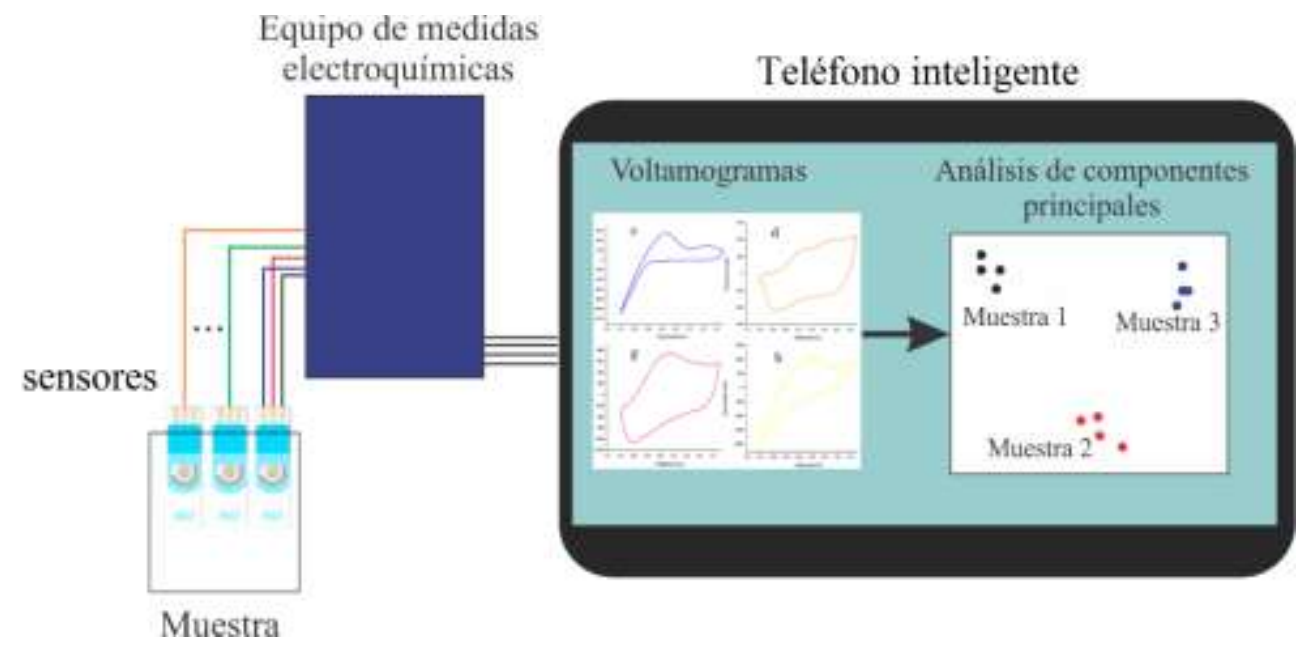

Fig. 1: Sistema utilizado para realizar las medidas con la mini-lengua electrónica y las redes de sensores bajo estudio

Como se mencionó anteriormente, la selectividad cruzada es otro aspecto importante a la hora de evaluar las capacidades de una red de sensores en la discriminación de muestras complejas. La selectividad cruzada consiste en que cada sensor debe responder de manera distinta frente a una muestra, asegurando así que cada uno aporta información útil para discriminarla y que la red de sensores registra señales distintas frente a cada una de las muestras, permitiendo que las señales recogen información de las diferencias fisicoquímicas entre las muestras. (González-Calabuig et al., 2016; Campos et al., 2013; Vagin y Winquist 2015; Arrieta et al., 2016; Díaz 2017). Esta particularidad es lo que permite que los dispositivos de lengua electrónica tengan la capacidad para clasificar y discriminar muestras complejas.

Para evaluar la selectividad cruzada, se realizaron los registros de las señales con cada una de las redes de sensores RS1 y RS2 frente a las 28 muestras; cuatro tipos de muestras de leches con siete réplicas de cada una. En la figura 2 se presentan algunas ejemplos de las señales voltamétricas registradas con sensores de las dos redes (RS1 y RS2). Los voltamogramas a, b y c, fueron registros realizados con los sensores DS-F10, DS-250-AT y DS-C013 de la RS 1 respectivamente (sensores comerciales serigrafiados con materiales electroactivos) y d, e y $\mathrm{f}$ registrados con los sensores PPy/PL, PPy/DBS y PPy/PSA de la red SR2, todos frente a una muestra de leche entera pasteurizada.

En ambos grupos de sensores, la variedad de picos anódicos y catódicos presente en los voltamogramas demuestran la capacidad de cada sensor para registrar y recoger información distinta de la muestra de leche y su composición compleja (proteínas, carbohidratos, lípidos, entre otros). (Bougrini et al., 2014; Sbodio et al., 2005; Sbodio et al., 2010). Las señales voltamétricas presentan diferencias apreciables en la forma; ancho de las curvas, intensidad de picos y potenciales de picos. Los potenciales de los picos se encuentran rotulados en cada una de las figuras y se observa la variedad valores obtenidos, las diferencias en las intensidades son evidenciadas en la escala de corriente de cada gráfica. Es de notar que para el resto de sensores, también se observaron perfiles voltamétricos diferenciados. Adicionalmente, los sensores en ambas redes de sensores presentaron una señal particular frente a cada una de las muestras, lo que contribuye a poder tener información para diferenciarlas. Estos resultados demuestran que las dos redes de sensores presentan selectividad cruzada frente a las muestras analizadas.

\section{Evaluación de la capacidad de discriminación y clasificación de las redes de sensores}

Después de verificar la respuesta de los sensores y su sensibilidad cruzada, se utilizó el análisis de componentes principales para evaluar su capacidad de discriminación y clasificación. Como se mencionó anteriormente, para evaluar la capacidad de discriminación de las redes de sensores RS1 y RS2, se realizaron medidas en 28 muestras de leche comercial (siete réplicas de cuatro tipos de muestras distintas). Luego de realizar las medidas como se detalla en el apartado experimental y ejecutando el análisis multivariante se obtuvieron las componentes principales para cada red de sensores. La figura 3 y 4 muestran el resultado del análisis de componentes principales correspondiente de las dos redes de sensores frente a muestras de leche entera pasteurizada, entera, semidescremada deslactosada y semidescremada. 

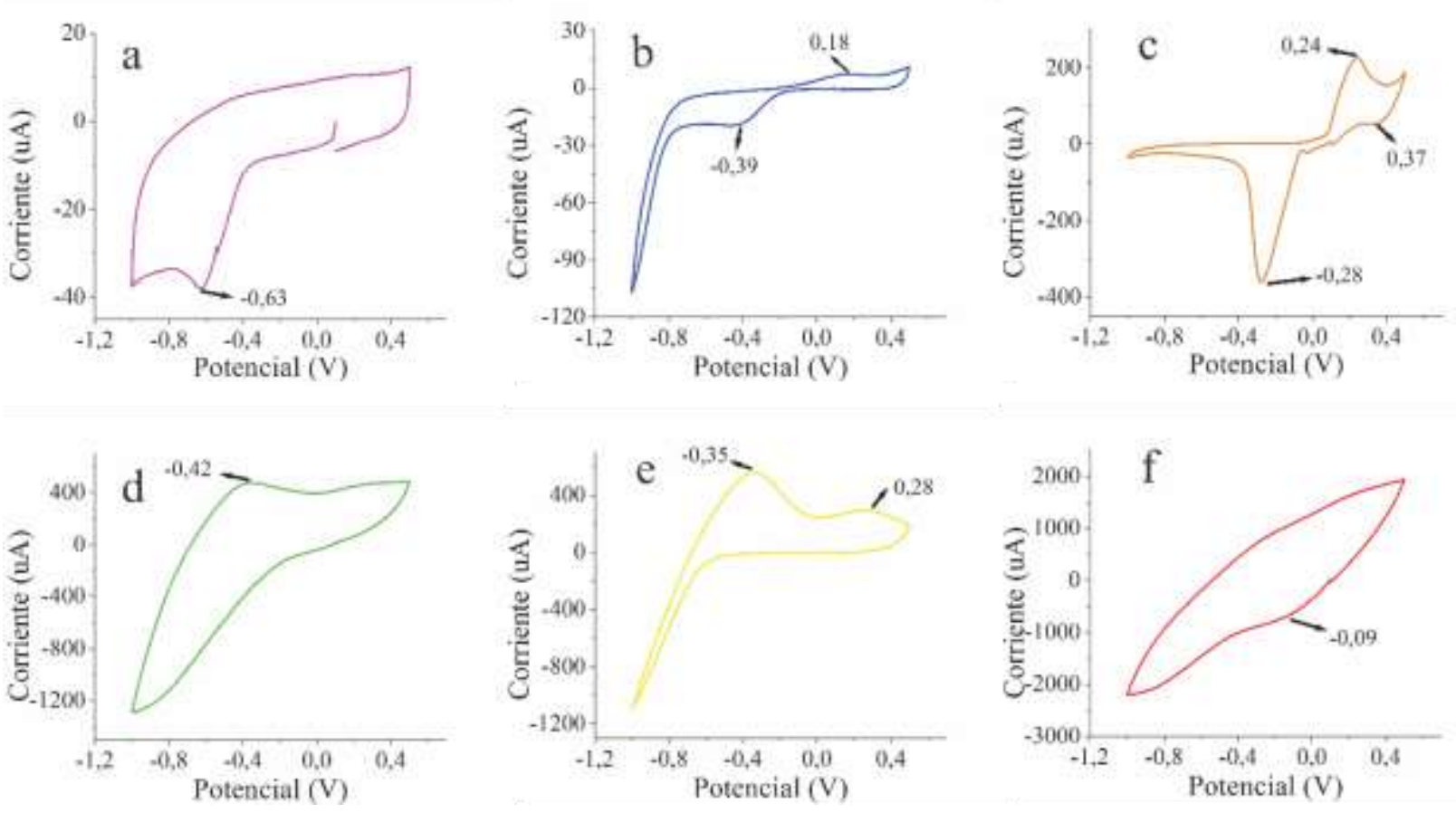

Fig. 2: Señales voltamétricas para RS1 (a) DS-F10, (b) DS-610, (c) DS-710 y RS2 (d) PPy/PL, (e) PPy/DBS, (f) PPy/PSA frente a una muestra de leche entera pasteurizada

En la figura 3, podemos observar que los sensores de la red RS1 son capaces de discriminar las muestras analizadas. Sin embargo, no recogen la información suficiente de las muestras para discriminarlas completamente, ya que existen puntos solapados de diferentes grupos de leche; como por ejemplo, el punto número 15 que pertenece a la muestra de leche semidescremada deslactosada tiende a solaparse con los de las muestras de leche entera pasteurizada, igualmente sucede con las réplicas 14 y 22 que corresponden a leche entera y semidescremada respectivamente, indicando una capacidad de discriminación limitada.

Por otra parte, para el mismo grupo de muestras de leche e iguales condiciones experimentales, el uso de la red de sensores modificados con polipirrol dopado con distintos contraiones permitieron obtener una discriminación mucho más clara de las muestras analizadas (figura 4). En la figura 4 se observan los grupos de muestras de leche discriminados por su naturaleza química; para el caso de la leche entera y entera pasteurizada se encuentran ubicadas en los cuadrantes positivos de la componente principal 1 (C.P.1) quizás debido a que ambos tipos de muestras poseen alto contenido de grasa butírica. En los cuadrantes negativos de la componente 1 , se encuentran ubicadas las muestras de leche semidescremada y semidescremada deslactosada, la particularidad de estas muestras radica a que debido a procesos industriales se da la reducción en su contenido de grasa butírica y de lactosa principalmente, lo que ofrece unas características fisicoquímicas similares en este tipo de muestras y que los diferencia de las muestras de leche entera.

Al comparar los valores de la varianza o información que se puede extraer en las dos primeras componentes principales de las matrices elaboradas con las señales de cada red de sensores, se obtuvo que para el caso de RS1 las CP1 y CP2 recogen un 56,50\% de información y para RS2 es de 67,64\%. Estos valores de información recolectada a partir de las señales en las dos primeras componentes, son coherentes con la capacidad de discriminación presentada por cada una de las redes de sensores, que para el caso de las red de sensores 1 fue menor que con la red de sensores 2. La diferencia en el valor de la varianza total, puede estar asociado a la electroactividad del polipirrol y los iones dopantes atrapados en la cadena de polipirrol, que para el caso de RS2 recoge información rica y variada del medio analizado, debido posiblemente a que la electroactividad del polipirrol se afecta por las sustancias (iónicas y no iónicas) circundantes que rodean la superficie del sensor, además algunas de las sustancias en la muestra pueden poseer electroactividad por sí misma y afectar el patrón de las señales aportando información para su discriminación. Por otra parte, en los sensores serigrafiados los materiales electroactivos que los constituyen no son afectados por intercambio iónico en su matriz como el polipirrol y esto puede reducir la cantidad y calidad de la información registrada en las señales. Además, el hecho de que con solo un $67,64 \%$ se pueda discriminar completamente las muestras estudiadas es indicativo de que la información registrada por los sensores poliméricos es información útil, debido a que en ella se capturan las particularidades de cada muestra y esto permite llevar a cabo la discriminación. 


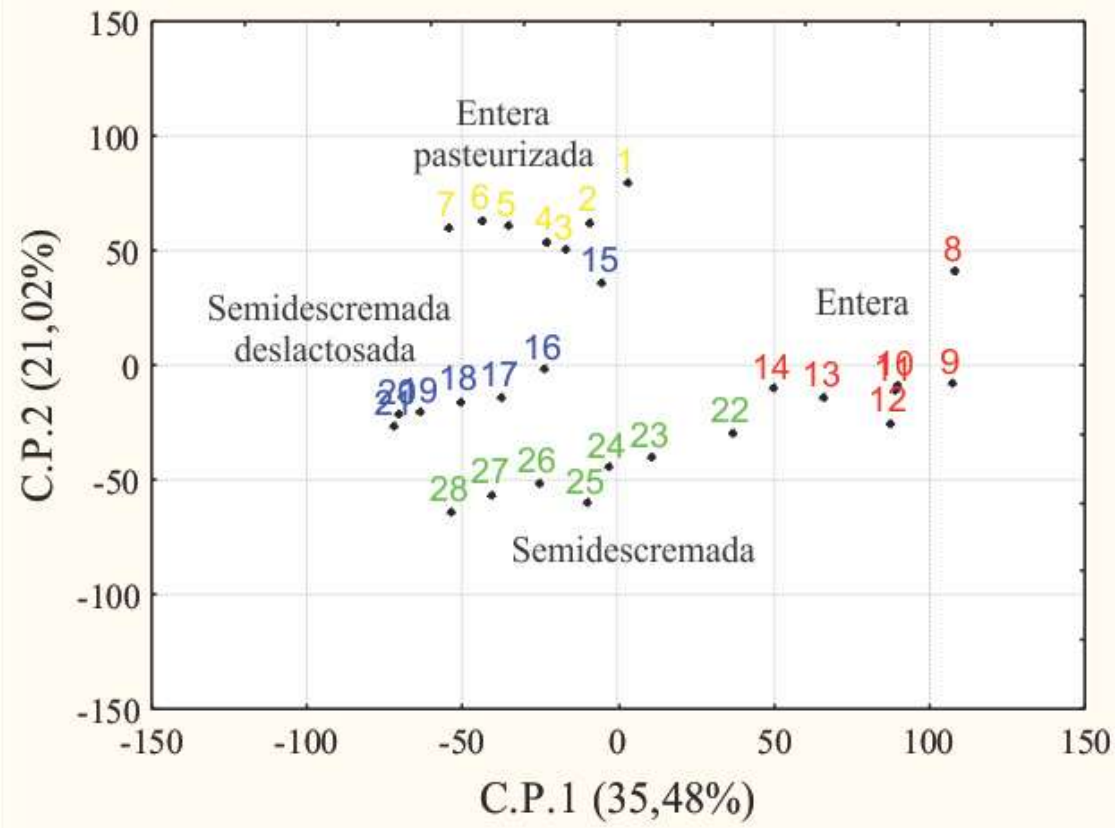

Fig. 3: Análisis de componentes principales para valores de corriente obtenidos por RS1 en muestras de leche comercial

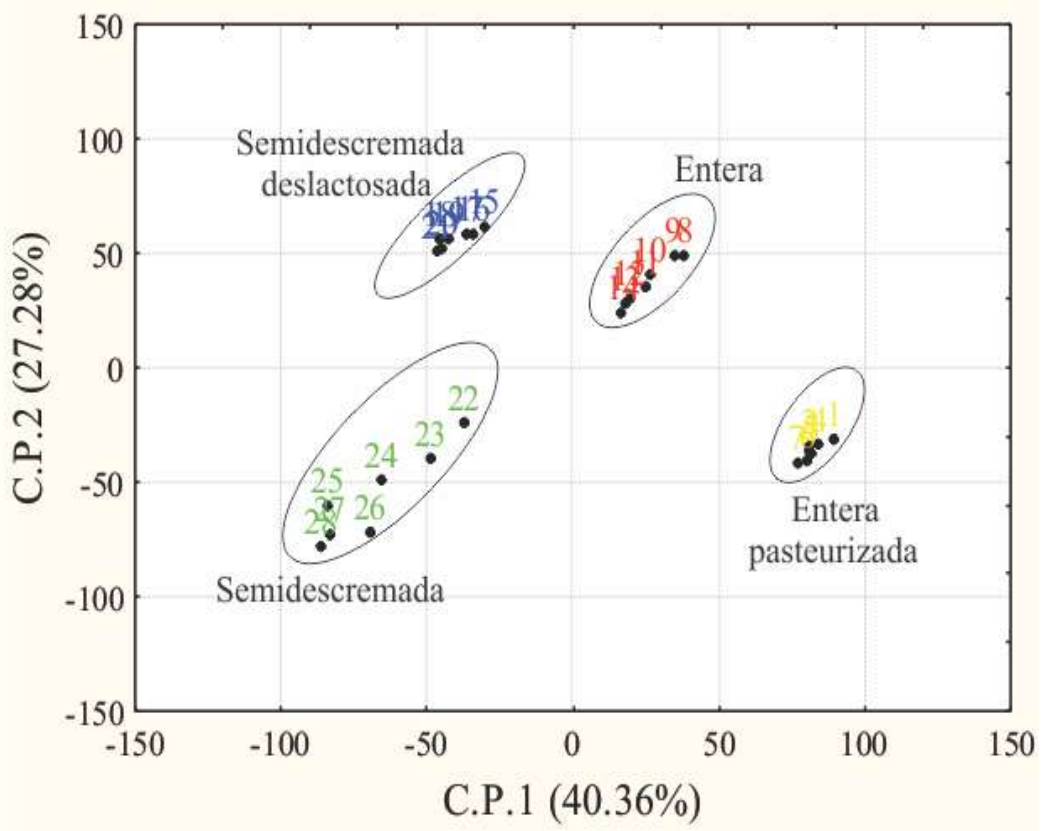

Fig. 4: Análisis de componentes principales para valores de corriente obtenidos por RS2 en muestras de leche comercial

Al realizar el análisis de componentes principales con SR1 y utilizar las tres primeras componentes principales; es decir, si se incorpora información adicional extraída por los sensores de la red RS1, se logra obtener dos agrupaciones de muestra claramente definidas y con una distribución espacial con las misma orientación obtenida con las dos primeras componentes del análisis realizado con la red RS2; las dos muestras de leche entera a un lado y las dos muestras de leche semidescremada al otro (figura 5). No obstante, nuevamente se observa que las puntuaciones 8 y 15 se localizan por fuera de sus grupos correspondientes. Este resultado muestra que los sensores de la red SR1 ofrecen menor información que la SR2 en las dos primeras componentes, y al incluir una componente adicional (CP3) la cual aumenta la cantidad de información a $72.18 \%$ pueden discriminar perfectamente las muestras con similar distribución espacial en concordancia con sus particularidades químicas y fisicoquímicas. 


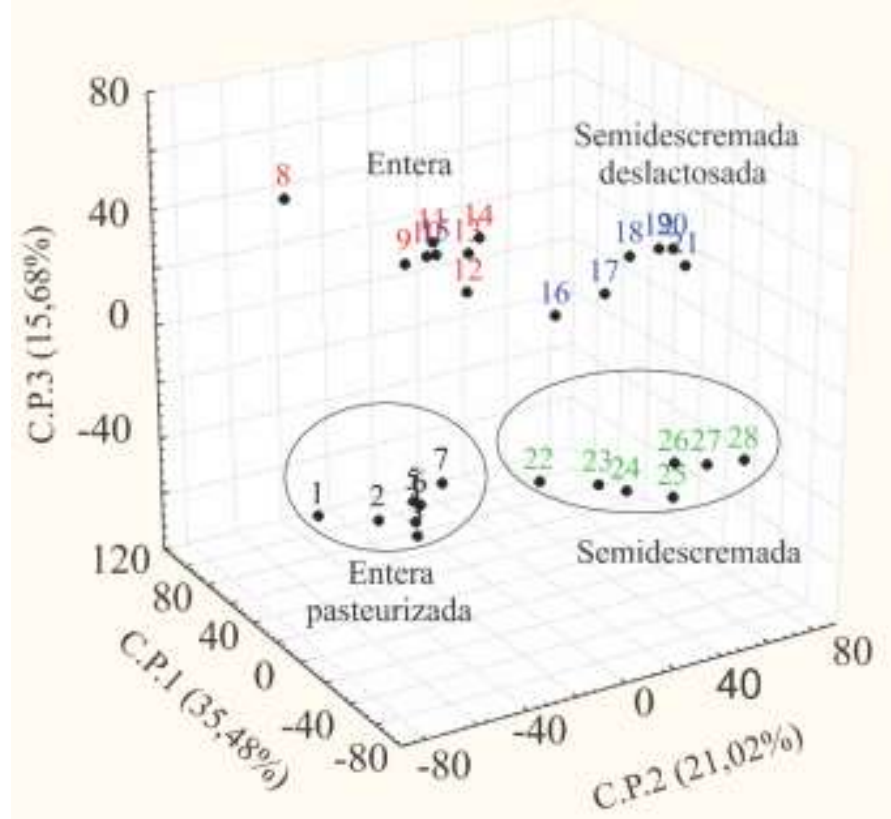

Fig. 5: Representación de las tres primeras componentes principales para valores de corriente captados por RS1

\section{CONCLUSIONES}

Las dos redes de sensores estudiadas presentan selectividad cruzada, lo que permite realizar la discriminación de las muestras. La modificación electroquímica con polipirrol sobre la superficie de los electrodos de trabajo permitió conseguir respuestas ricas en información; aportando así a una buena clasificación de muestras con diferentes propiedades fisicoquímicas y organolépticas.

El método de reconocimiento de patrones implementado (PCA) ofrece correctamente una discriminación y clasificación de los valores de corriente, presentando una excelente capacidad para el reconocimiento de patrones en muestras complejas como la leche.

La red de sensores compuesta por sensores poliméricos de polipirrol ofrece mayor estabilidad e información de las muestras analizadas permitiendo discriminarlas con solo dos componentes. En el caso de la red de sensores serigrafiados con materiales electroactivos es capaz de discriminar las muestras en su totalidad utilizando tres componentes.

\section{AGRADECIMIENTOS}

Los autores agradecen el soporte financiero brindado por COLCIENCIAS (contrato $N^{\circ}$ : 0401-2013), y la Universidad Pontificia Bolivariana seccional Montería.

\section{REFERENCIAS}

Amin, O., A. Arrieta, Lengua electrónica portátil para el análisis de leche cruda basada en tecnología PSoC (Programmable System on Chip) y Android, Ingeniare Revista Chilena de Ingeniería, 24, 445-453 (2016)

Arrieta, A., J. Díaz, O. Amin, Elaboración y optimización de una red de sensores electroquímicos para una lengua electrónica orientada al análisis de leche, Rev. Soc. Quím. Perú, 82, 152-161 (2016)

Blanco, C.A., R. De la Fuente, I. Cabellero, M.L. Rodríguez-Méndez, Beer discrimination using a portable electronic tongue based on screen-printed electrodes, J. Food Eng., 157, 57-62 (2015)

Bougrini, M., K. Tahri, Z. Haddi, N. El Bari, E. Llobet, N. Jaffrezic-Renault, B. Bouchikhi, Aging time and brand determination of pasteurized milk using a multisensor e-nose combined with a voltammetric e-tongue, Mater Sci. and Eng. C., 45, 348-358 (2014)

Campos, I., R. Bataller, R. Armero, J.M. Gandia, J. Soto, R. Marínez-Máñez, Monitoring grape ripeness using a voltammetric electronic tongue, Food Res. Int., 54, 1369-1375 (2013) 
Cetó, X., J.M. Gutiérrez, M. Gutiérrez, F. Céspedes, J. Capdevila, S. Mínguez, C. Jiménez, M. Del Valle, Determination of total polyphenol index in wines employing a voltammetric electronic tongue, Anal Chim. Acta, 732 (30), 172-179 (2012)

Ciosek, P., Wroblewski, W., Miniaturized electronic tongue with an integrated reference microelectrode for the recognition of milk samples, Talanta, $76,548-556$ (2008)

De Saja, J.A., A. Arrieta, M.L. Rodríguez, V. Parra, J.A. Fernandez-Escudero, Electronic tongue based on chemically modified electrodes and voltammetry for the detection of adulterations in wines, Sensor Actuat BChem., 118 (1-2), 448-453 (2006)

Dias, L.A., A.M. Peres, A.C.A. Veloso, F.S. Reis, M. Vilas-Boas, A. Machado, An electronic tongue taste evaluation: Identification of goat milk adulteration with bovine milk, Sensor Actuat B-Chem., 136 (1-2), 209217 (2009)

Díaz, J., Desarrollo de una red de sensors basados en polipirrol y su aplicación en la discriminación de muestras de leche cruda contaminadas con pesticidas, Tesis de Maestría, Facultad de Ciencias, Universidad de Córdoba (2017)

Elizabeth, A. B., Jinhe B., Anne P., Sharon, D., Electronic Noses and Tongues: Applications for the Food and Pharmaceutical Industries, Sensors, 11(5), 4744-4766 (2011)

González-Calabuig, A., X. Cetó, M. del Valle, Electronic tongue for nitro and peroxide explosive sensing, Talanta, 153 (1), 340-346 (2016)

Gutiérrez, J.M., Z. Haddi, A. Amari, B. Benachir, A. Mimendia, X. Cetó, M. Del Valle, Hybrid electronic tongue base don multisensor data fusion for discrimination of beers, Sensor Actuat B-Chem., 177, 989-996 (2013)

Hruskar, M., Major, N., Krpan, M., Application of a potentiometric sensor array as a technique in sensory analysis, Talanta, 81, 398-403 (2010)

Huynh, T., W. Kutner, Molecularly imprinted polymers as recognition materials for electronic tongues, Biosens Bioelectron, 74, 856-864 (2015)

Lu, L., X. Hu, S. Tian, S. Deng, Z. Zhu, Visualized attribute analysis approach for characterization and quantification of rice taste flavor using electronic tongue, Anal Chim. Acta, 919 (5), 11-19 (2016)

Mari Luz, R., Electronic noses and tongues in food science, pp. 10-24, Academic Press, $1^{\text {a }}$ Ed., U.S.A. (2016)

Novakowsky, W., M. Bertotti, T.R.L.C. Paixão, Use of copper and gold electrodes as sensitive elements for fabrication of an electronic tongue: Discrimination of wines and whiskies, Microchem. J., 99(1), 145-151 (2011)

Paixão, T.R.L.C., M. Bertotti, Fabrication of disposable voltammetric electronic tongues by using Prussian Blue films electrodeposited onto CD-R gold surfaces and recognition of milk adulteration, Sensor Actuat B-Chem., 137 (1), 266-273 (2009)

Rahaman-Khan, M.R., S. Kang, Highly sensitive multi-channel IDC sensor array for low concentration taste detection, Sensors, 15, 13201-13221 (2015)

Sbodio, O., Tercero, E., Coutaz, V.R., Revelli, G., Propiedades de los geles lácteos formados por la acción combinada de enzimas coagulantes y glucono- $\delta$-lactona, Información Tecnológica, 16(4), 17-25 (2005)

Sbodio, O., Tercero, E., Zannier, M., Revelli, G., Tratamiento térmico de leche: influencia del pH y $\mathrm{CaCl}_{2}$ en la elaboración de queso cuartirolo, Información Tecnológica, 21(5), 107-116 (2010)

Vagin, M.Y., F. Winquist, Electronic noses and tongues in food safety assurance, in High Throughput Screening for Food Safety Assessment, pp. 266-283, Woodhead Publishing, U.S.A. (2015)

Wei, Z., J. Wang, X. Zhang, Monitoring of quality and storage time of unsealed pasteurized milk by voltammetric electronic tongue, Electrochim. Acta, 88, 231-239 (2013)

Wey, Z., J. Wang, Detection of antibiotic residues in bovine milk by a voltammetric electronic tongue system, Anal Chim. Acta, 694, 46-56 (2011) 